\title{
Comparative ability of dobutamine and exercise stress in inducing myocardial ischaemia in active patients
}

T H Marwick, A M D'Hondt, G H Mairesse, T Baudhuin, W Wijns, J M Detry,
J A Melin
(NS). Normal perfusion was identified in $70 \%$ of these patients at exercise MIBI, and $68 \%$ at dobutamine stress (NS).

Conclusions-In a group of patients studied under normal clinical circumstances antianginal treatment and inability to complete the stress protocol are frequent and compromise the capacity of dobutamine stress to induce ischaemia. In contrast, the induction of perfusion heterogeneity is less susceptible to submaximal stress.

(Br Heart f 1994;72:31-38) methoxybutylisonitrile (MIBI) single photon emission computed tomography. Main outcome measure-Coronary disease defined as an ST segment depression of $\geqslant 0.1 \mathrm{mV}$, a resting or stress induced perfusion defect, or a resting or stress induced wall motion abnormality on exercise and dobutamine stress testing. Results-Dobutamine stress was submaximal in 51 patients because of ingestion of $\beta$ adrenoceptor blocking agents on the day of the test $(n=25)$ or failure to attain the peak dose owing to side effects $(n=28)$. Exercise was limited in 23 patients by non-cardiac symptoms. The peak heart rate with dobutamine was less than that attained with exercise (105 (25) $v 132$ ( 24) beats/min, $P<0.0001$ ); the response to maximal dobutamine stress significantly exceeded that to submaximal stress. Peak blood pressure was greatest with exercise (206 ( 27) $v 173$ (25) mm $\mathrm{Hg}, \mathrm{P}<0.001$ ), values at maximal and submaximal dobutamine stress being comparable. Electrocardiographic evidence of ischaemia was induced less frequently by dobutamine than exercise (32\% $v 77 \%$ of the 56 patients with significant coronary disease, $P<0.01$ ), as was abnormal wall motion (54\% $v 88 \%$, $P<0.001)$. Ischaemia was induced more readily with maximal stress of either type; thus the sensitivities of dobutamine and exercise echocardiography were comparable only in patients undergoing a maximal dobutamine testing (73\% v $77 \%$, NS). Perfusion heterogeneity was induced in $\mathbf{5 8 \%}$ of patients with coronary disease at submaximal dobutamine stress, $73 \%$ at maximal dobutamine stress, and $73 \%$ at exercise stress (NS). Among 30 patients without coronary stenoses, normal function was obtained in $83 \%$ of echocardiography studies with dobutamine and in $80 \%$ with exercise
Regional abnormalities in perfusion or wall motion that are not apparent at rest may be induced by increasing cardiac workload in patients with coronary artery disease. ${ }^{1}$ The optimal means of increasing cardiac work for the induction of ischaemia has not been defined, although exercise is accepted as the preferred stress test in patients who are able to exercise maximally. ${ }^{2}$ The combination of echocardiography with exercise is, however, technically difficult both to perform and to interpret-something that has been ameliorated but not solved by image digitisation. ${ }^{3}$

Dobutamine stress testing may be combined with either echocardiography ${ }^{4-8}$ or perfusion scintigraphy ${ }^{910}$ to detect coronary artery disease in patients who are unable to exercise. Because exercise induced hyperventilation does not occur, and the patient remains stationary during the test, image acquisition is easier; the availability of better quality images may also make interpretation easier. These considerations have made dobutamine echocardiography an attractive alternative to exercise echocardiography, even in patients who can exercise maximally. However, the recorded sensitivities of stress echocardiography using dobutamine $e^{4-8}$ and exercise vary ${ }^{11-16}$ according to the study population and clinical state. The techniques need to be compared under the same circumstances, and the comparative influence of factors such as antianginal drug ingestion and submaximal stress-which may compromise either test, but to various extents-have not been clarified. We compared the ability of exercise and dobutamine stress to increase cardiac workload and induce ischaemia or flow heterogeneity in the same heterogeneous population of patients undergoing coronary angiography under routine clinical circumstances. In addition, we assessed the
Dr T Marwick, Department of Cardiology, Cleveland Euclid Avenue, Cleveland OH 44195, USA.

Accepted for publication 20 December 1993 
feasibility of each method for stress echocardiography by comparing the quality of echocardiograms and their ease of interpretation.

\begin{abstract}
Methods
PATIENTS

Eighty six patients without a history of myocardial infarction, $(60 \mathrm{men}$ and 26 women, mean age 59 (9) years) were studied at the time of diagnostic coronary angiography for symptoms suggestive of coronary artery disease. Dobutamine and exercise stress tests were both performed during the patients' hospital admission for coronary angiography, and the tests were therefore separated by 1-2 days. Tests were performed in random order, except when the second test was arranged after angiography for scheduling reasons, in which case dobutamine echocardiography was performed at this time because the arterial wound limited the ability to exercise maximally. Patients with contraindications to dobutamine or exercise stress testing (principally unstable angina, serious arrhythmias, and severe hypertension) were excluded for safety reasons. Patients with severe valvar heart disease, cardiomyopathy, or previous coronary bypass grafting were also excluded because correlating the results of stress testing with those of angiography is limited under these circumstances. The study protocol was approved by the hospital ethics committee.
\end{abstract}

CORONARY ANGIOGRAPHY

Selective coronary angiograms were obtained in multiple projections. The films were reviewed by an independent observer blinded to the clinical features, electrocardiographic, echocardiographic, and scintigraphic results. Stenoses were traced manually, and the percentage of stenosis was calculated by comparing the stenosis diameter with an adjacent normal segment. Significant coronary stenoses (defined by $>50 \%$ reduction in luminal diameter) were present in 56 patients, 34 of whom had multivessel disease.

\section{EXERCISE STRESS}

Patients exercised using a previously described cycle ergometer protocol, ${ }^{17}$ starting at $20 \mathrm{~W}$ and increasing in increments of $20 \mathrm{~W}$ every minute. Patients were encouraged to perform maximal exercise; clinical, electrocardiographic, and echocardiographic features were monitored throughout the test. Exercise was limited by symptoms, being stopped for severe angina $(n=18)$, hypotension $(n=11)$, and non-ischaemic end points, including dyspnoea $(n=13)$, fatigue $(n=42)$, and hypertension $(n=2)$.

\section{DOBUTAMINE STRESS}

Dobutamine was administered by continuous intravenous injection using a previously described protocol. ${ }^{10}$ The starting dose was $5 \mu \mathrm{g} / \mathrm{kg} /$ minute, and was increased in increments every three minutes to $10,20,30$, and $40 \mu \mathrm{g} / \mathrm{kg} /$ minute. The test was monitored in the same way as the exercise tests. The study was terminated at the end of the protocol in 50 patients, because of severe angina in eight, and because of side effects in 28 . Side effects comprised hypotension $(>20 \mathrm{~mm} \mathrm{Hg}$ reduction in blood pressure) in 20 patients, hypertension (systolic blood pressure $>220$ $\mathrm{mmHg}$ ) in three patients, arrhythmia (sustained supraventricular tachycardia, non-sustained ventricular tachycardia, and frequent multifocal ventricular extrasystoles) in three patients, and intolerable symptoms of anxiety in two patients. All side effects were self limiting, and no serious complications occurred.

ELECTROCARDIOGRAPHIC ANALYSIS

The Frank leads $\mathrm{X}, \mathrm{Y}$, and $\mathrm{Z}$ were monitored continuously and digitised each minute during each stress test. ${ }^{17}$ Blood pressure and pulse rates were recorded at the conclusion of each dobutamine dose, and every two minutes during exercise stress testing. Electrocardiograms were read by an independent observer blinded to the angiographic and echocardiographic data. Significant ST segment change was defined as an ST depression of more than $0.1 \mathrm{mV}$ or ST elevation at $0.06 \mathrm{~ms}$ after the $\mathrm{J}$ point.

\section{ECHOCARDIOGRAPHY}

Cross sectional echocardiography was performed using standard equipment, and in the four standard views. The heart was imaged continually during stress testing, with recordings at the end of each three minute increase in dobutamine dose and every two minutes during exercise stress testing. Imaging was continued for three minutes at the end of testing or until abnormalities in wall motion resolved. In addition to recording on half inch video tape, images were digitised on line and portrayed in a split screen (four screens). Images while the subject was at rest were compared with images at the peak of exercise or after exercise or with images at low (10 $\mu \mathrm{g} / \mathrm{kg} / \mathrm{min}$ ) and high doses (30 and 40 $\mu \mathrm{g} / \mathrm{kg} / \mathrm{min}$ ) of dobutamine.

Echocardiographic data were interpreted by the two observers blinded to the clinical, electrocardiographic, and angiographic features of the patients by means of digitised images and video recordings. Interpretations were performed using a semi quantitative approach, ${ }^{18}$ which entailed comparing regional wall motion in 16 myocardial segments divided according to the criteria of the American Society of Echocardiography. These segments were defined to match the territories of the coronary arteries as previously described ${ }^{14}$; the anterior, apical, septal, and anteroseptal walls were ascribed to the left anterior descending artery, the posterior and lateral walls to the left circumflex artery, and the inferior and basal septal segments to the right coronary artery. Each region was scored 1 (normal), 2 (hypokinetic), 3 (akinetic), or 4 (dyskinetic). A normal response in either test comprised homogeneous contraction at rest and with stress. Myocardial ischaemia was identified by 
the development of regional hypokinesis, akinesis, or dyskinesis in response to stress. Myocardial infarction (present as the only abnormality in only two patients because of the selection criteria) was identified by resting akinesis or dyskinesis. Hypokinesis at rest was identified as normal if it improved in parallel with the rest of the myocardium, or ischaemic if it deteriorated or failed to improve in the presence of enhanced regional wall motion elsewhere. These criteria were used to classify echocardiograms as normal or abnormal.

In addition to the categorisation of normal and abnormal responses, the extent of ischaemia was defined by the proportion of the 16 segments which showed ischaemia. The feasibility of each stress technique for echocardiography was compared on the grounds of image quality and ease of interpretation. Image quality was described using a previously defined four point score, whereby an A quality image showed the complete endocardium, a B quality image showed all segments without complete endocardial definition, a C quality image did not permit detection of one or more segments, but adjacent segments within the same coronary territory could be interpreted in other views, and a D quality study precluded interpretation of segments comprising one or more territories. ${ }^{14}$ Ease of interpretation was defined subjectively, using a continuous three point score. Finally, studies were read independently from tape and digitised images so that sensitivity and specificity were obtained for each recording method.

\section{PERFUSION IMAGING}

Technetium-99m methoxybutylisonitrile (20 $\mathrm{mCi}$ ) was injected 1 to 2 minutes before conclusion of exercise stress in all 86 patients and in 82 dobutamine studies. Stress perfusion imaging was performed 1 to 2 hours later. The same dose of isotope was injected at rest on another day to obtain resting images. If resting images were required on the same day because of scheduling considerations, the resting scan (using only $5 \mathrm{mCi}$ of the agent) was performed first, with stress imaging six hours later. ${ }^{19}$ Scintigraphic data were acquired using a large field, single crystal camera and high resolution collimator (General Electric $400 \mathrm{AC} / \mathrm{T}$ ), over a $180^{\circ}$. Transaxial images were obtained by back projection using a Shepp-Logan filter and then reoriented into vertical and horizontal long and short axis views.

Perfusion scintigraphy was interpreted by experienced observers blinded to the other investigations. The same segmentation as that used for echocardiography was used to permit a qualitative comparison between stress and

Table 1 Mean (SD) peak heart rate, systolic blood pressure, and rate-pressure product in 86 patients on dobutamine and exercise stress testing

\begin{tabular}{llll}
\hline & Heart rate (beats/min) & Systolic pressure $(\mathrm{mm} \mathrm{hg})$ & Rate of pressure product \\
\hline Dobutamine & $106(25)$ & $174(25)$ & $185(55)$ \\
Exercise & $133(23)$ & $206(27)$ & $271(68)$ \\
P value & 0.001 & 0.0001 & 0.0001 \\
\hline
\end{tabular}

rest images. An analogous defect extent score was derived by expressing the number of abnormal segments as a percentage of the total. Regions were interpreted as showing normal perfusion, a stress induced perfusion defect, or a fixed perfusion defect (eight patients).

\section{DATA ANALYSIS}

The results of exercise and dobutamine stress echocardiography were compared as paired data by McNemar's test. Continuous variables for exercise and dobutamine stresses were compared using paired $t$ tests. Unpaired data were compared using the $\chi^{2}$ test, with Yates's correction or Fisher's exact test, depending on the sample size. Results were expressed as means (SD), and significant differences were defined as $P<0.05$.

\section{Results}

RESPONSES TO DOBUTAMINE AND EXERCISE STRESS

Table 1 shows the responses of all 86 patients to dobutamine and exercise stress testing. In the overall group dobutamine stress induced a significantly lower peak heart rate, peak blood pressure, and rate-pressure product than exercise. The augmentation of heart rate with dobutamine stress was 38 (21) beats/minute compared with 60 (25) beats/minute with exercise, and the augmentation of blood pressure was 28 (23) $\mathrm{mm} \mathrm{Hg}$ compared with 51 (27) $\mathrm{mm} \mathrm{Hg}$ with exercise. Maximal exercise stress (defined by attainment of $>85 \%$ age predicted maximal heart rate) was performed in 63 patients and submaximal stress in 23 patients $(27 \%)$. In contrast, only 10 patients developed ischaemia or attained $>85 \%$ of age predicted maximum heart rate with dobutamine $(\mathbf{P}=0.02 v$ exercise $)$.

Thirty five patients completed a maximal dobutamine protocol-that is, developed ischaemia or achieved the maximum dose and were not taking $\beta$ adrenoceptor blocking drugs - and 51 (59\%) had a submaximal test (based on premature termination of the protocol or ingestion of $\beta$ adrenoceptor blocking drugs on the day of the test). In patients undergoing a maximal dobutamine stress the maximum attained heart rate exceeded that at submaximal stress (117 (20) $v 98$ (26) beats/minute, $P<0.001$ ) but remained less than that attained with exercise $(P=0.005)$. Maximum blood pressures were the same in patients with maximal and submaximal dobutamine stress (173 (20) $v 174(28) \mathrm{mm} \mathrm{Hg}$ ) and were lower than those recorded at exercise stress $(P<0.0001)$.

ISCHAEMIC ELECTROCARDIOGRAPHIC RESPONSES WITH DOBUTAMINE AND EXERCISE Table 2 shows the development of electrocardiographic changes or angina, or both in response to dobutamine or exercise for various subgroups. Among the 56 patients with coronary disease, ischaemia (defined by either angina or ST segment depression) was identified less frequently during dobutamine 
Table 2 Electrocardiographic responses during maximal (P value) and submaximal exercise and dobutamine stress testing

\begin{tabular}{lcccc}
\hline & All patients & Maximal & Submaximal & $\begin{array}{c}\text { Maximal v } \\
\text { submaximal }\end{array}$ \\
\hline Sensitivity & $43 / 56(77 \%)$ & $39 / 44(91 \%)$ & $4 / 12(25 \%)$ & $0 \cdot 0003$ \\
Specificity & $13 / 30(43 \%)$ & $4 / 19(21 \%)$ & $9 / 11(82 \%)$ & 0.002 \\
Accuracy & $56 / 86(65 \%)$ & $43 / 63(68 \%)$ & $13 / 23(57 \%)$ & $\mathrm{NS}$ \\
& & Dobutamine & $5 / 34(15 \%)$ & $<0 \cdot 001$ \\
Sensitivity & $18 / 56(32 \%)$ & $13 / 22(59 \%)$ & $12 / 17(71 \%)$ & $\mathrm{NS}$ \\
Specificity & $19 / 30(67 \%)$ & $7 / 13(54 \%)$ & $17 / 51(33 \%)$ & 0.02 \\
Accuracy & $37 / 86(43 \%)$ & $20 / 35(57 \%)$ & $17 \%)$ \\
\hline
\end{tabular}

Table 3 Frequency of ischaemia (\%) evidenced by electrocardiographic changes or angina, echocardiographic wall motion abnormalities, and regional perfusion defect on perfusion scintigraphy in patients undergoing maximal and submaximal dobutamine stress testing

\begin{tabular}{|c|c|c|c|}
\hline & $\begin{array}{l}\text { Electrocardiographic } \\
\text { changes/angina }\end{array}$ & $\begin{array}{l}\text { Echocardiographic wall } \\
\text { motion abnormalities }\end{array}$ & $\begin{array}{l}\text { Regional perfusion } \\
\text { defect on scintigraphy }\end{array}$ \\
\hline $\begin{array}{l}\text { Dobutamine } \\
\text { Exercise } \\
\text { P value }\end{array}$ & $\begin{array}{l}\text { Patients undergoing } n \\
59 \\
77 \\
\text { NS }\end{array}$ & $\begin{array}{l}\text { aximal dobutamine stress ( } \\
73 \\
77 \\
\text { NS }\end{array}$ & $\begin{array}{l}=22) \\
76 \\
67 \\
\text { NS }\end{array}$ \\
\hline $\begin{array}{l}\text { Dobutamine } \\
\text { Exercise } \\
\text { P value }\end{array}$ & $\begin{array}{l}\text { Patients undergoing } \\
15 \\
68 \\
0.0001\end{array}$ & $\begin{array}{l}\text { bmaximal dobutamine stres } \\
41 \\
94 \\
0.0001\end{array}$ & $\begin{array}{l}(n=34) \\
58 \\
79 \\
0.02\end{array}$ \\
\hline
\end{tabular}

compared with exercise stress $(32 \% v 77 \%, \mathrm{P}$ $<0.0001)$. This difference pertained both to the 32 patients with multivessel disease $(29 \% v$ $71 \%, \mathrm{P}<0.001)$ and to those with single vessel disease $(36 \% v 73 \%, P=0.02)$.

Among the 22 patients with coronary disease who underwent maximal dobutamine stress angina or ST segment changes occurred with comparable frequency during dobutamine and exercise testing (table 3 ). In the remaining patients with coronary stenoses who underwent submaximal dobutamine stress, however, ischaemic changes were significantly less common during dobutamine than exercise stress $(15 \% v 68 \%, \mathrm{P}<0.0001)$

Table 4 Frequency of ischaemia (\%) evidenced by electrocardiographic changes or angina, echocardiographic wall motion abnormality, and regional perfusion defect or perfusion scintigraphy in patients undergoing maximal and submaximal exercise testing

\begin{tabular}{llll}
\hline & $\begin{array}{l}\text { Electrocardiographic } \\
\text { changes/angina }\end{array}$ & $\begin{array}{l}\text { Echocardiographic wall } \\
\text { motion abnormalities }\end{array}$ & $\begin{array}{l}\text { Regional perfusion } \\
\text { defect or scintigraphy }\end{array}$ \\
\hline Dobutamine & Patients undergoing & maximal exercise stress $(n=44)$ \\
Exercise & 36 & 59 & 66 \\
P value & 89 & 93 & 75 \\
& 0.0001 & 0.001 & $\mathrm{NS}$ \\
Dobutamine & Patients undergoing submaximal exercise stress $(n=12)$ \\
Exercise & 15 & 33 & 60 \\
P value & 25 & 67 & 67 \\
& NS & NS & NS
\end{tabular}

Table 5 Echocardiographic abnormalities in wall motion during maximal and submaximal exercise and dobutamine stress testing

\begin{tabular}{lrrrl}
\hline & All patients & Maximal & Submaximal & $\begin{array}{l}\text { Maximal } v \\
\text { submaximal }\end{array}$ \\
\hline Sensitivity: & & Exercise & & \\
Overall & $49 / 56(88 \%)$ & $41 / 44(93 \%)$ & $8 / 12(67 \%)$ & $0 \cdot 03$ \\
Single vessel disease & $18 / 22(82 \%)$ & $16 / 18(89 \%)$ & $2 / 4(50 \%)$ & NS \\
Multivessel disease & $31 / 34(97 \%)$ & $25 / 26(96 \%)$ & $6 / 12(50 \%)$ & $0 \cdot 002$ \\
Specificity & $24 / 30(80 \%)$ & $15 / 19(79 \%)$ & $9 / 11(82 \%)$ & NS \\
Accuracy & $73 / 86(85 \%)$ & $56 / 63(89 \%)$ & $17 / 23(74 \%)$ & NS \\
& & Dobutamine & & \\
Sensitivity: & & & \\
Overall & $30 / 56(54 \%)$ & $16 / 22(73 \%)$ & $14 / 34(41 \%)$ & $0 \cdot 02$ \\
Single vessel disease & $8 / 22(36 \%)$ & $5 / 10(50 \%)$ & $3 / 12(25 \%)$ & NS \\
Multivessel disease & $22 / 34(65 \%)$ & $11 / 12(92 \%)$ & $11 / 22(50 \%)$ & $0 \cdot 02$ \\
Specificity & $25 / 30(83 \%)$ & $12 / 13(92 \%)$ & $13 / 17(76 \%)$ & NS \\
Accuracy & $55 / 86(64 \%)$ & $28 / 35(80 \%)$ & $27 / 51(53 \%)$ & $0 \cdot 01$ \\
\hline
\end{tabular}

(table 3). Similarly, in the 44 patients with coronary disease who were able to exercise maximally ischaemic changes occurred more frequently during exercise than dobutamine stress $(89 \% v 36 \%, \mathrm{P}<0.0001)$, but in those unable to achieve maximal exercise ischaemia was rarely induced by either stress (table 4 ).

DETECTION OF ABNORMAL REGIONAL FUNCTION DURING DOBUTAMINE AND EXERCISE

Table 5 shows the frequency of disturbances of regional function in response to dobutamine and exercise. Of the 56 patients with coronary stenoses, $30(54 \%)$ had abnormal wall motion with dobutamine and $49(88 \%)$ at peak exercise $(P=0.0002 v$ dobutamine $)$. The images after exercise were less sensitive than those at peak stress, detecting 38 patients ( $69 \%, P=0.004 v$ peak).

Thirty four patients had multivessel coronary disease, and in this group the sensitivity of peak exercise imaging also exceeded that of imaging after exercise $(91 \%$ v $74 \%, \mathrm{P}=$ $0.04)$. Dobutamine echocardiography had a lower sensitivity in this group than peak exercise $(65 \%, P=0.02)$, although 11 of the 12 patients $(92 \%)$ with multivessel disease undergoing a maximal dobutamine stress were identified by dobutamine echocardiography (NS $v$ exercise). The ability of each test to induce ischaemia in the 22 patients with single vessel coronary disease was less than that in patients with multivessel disease, although this attained significance for only dobutamine echocardiography $(36 \% v 65 \%, P=0.05)$. Abnormal function in patients with single vessel disease was induced in $82 \%$ at peak exercise and $67 \%$ after exercise (NS). Abnormal wall motion was more frequently detected in this group at either peak exercise or after exercise at dobutamine echocardiography (36\%, $P=0.01$ ), although this difference was less obvious in patients with a maximal dobutamine stress (sensitivity $50 \%, P=0.08$ ).

Ischaemia was not induced by dobutamine, despite the presence of coronary disease, in patients taking $\beta$ adrenoreceptor blocking drugs less than 12 hours before the test and in those unable to complete the stress protocol. Of 26 patients with false negative results in dobutamine echocardiograms, $13(50 \%)$ had taken a $\beta$ blocker on the day of the test, compared with seven of the 30 with true positive results $(23 \%, P=0.05)$. Premature termination of dobutamine stress because of side effects accounted for $42 \%$ of false negative results in dobutamine echocardiograms (compared with patients achieving peak dose accounting for $28 \%$ of false negative results). Among patients with significant coronary disease who underwent submaximal dobutamine stress (table 3), echocardiographic evidence of ischaemia was induced less commonly by dobutamine than exercise $(41 \% \quad v \quad 94 \%$, $\mathrm{P}<0.0001)$. In contrast, among the 22 patients with coronary disease at maximal dobutamine stress (table 3) abnormal regional function was induced in a comparable proportion of studies with dobutamine and exercise 
$(73 \% v 77 \%)$. In summary, the overall sensitivity of exercise echocardiography for the detection of ischaemia exceeded that of submaximal but not maximal dobutamine echocardiography.

The performance of submaximal exercise (termination of stress at $<85 \%$ age predicted maximum heart rate in the absence of ischaemic end points) was associated with a lower sensitivity of exercise echocardiography (table 5). Indeed, only in those who exercised maximally did the sensitivity of exercise echocardiography exceed that of dobutamine echocardiography (table 4). The inability of some patients to achieve maximal heart rates reflected the recent ingestion of $\beta$ blockers in 15 patients $(68 \%)$, a factor present in only 16 of the 63 patients performing maximal exercise $(25 \%, P<0.001)$. Twenty patients with coronary disease took $\beta$ blockers on the day of the exercise test; of six false negative results on exercise echocardiography, four (67\%) patients had taken $\beta$ blockers, compared with 17 of the 50 patients with true positive results $(34 \%, N S)$.

\section{PERFUSION HETEROGENEITY WITH \\ DOBUTAMINE AND EXERCISE}

Table 6 summarises the relative abilities of dobutamine and exercise stress to induce perfusion heterogeneity. Regional perfusion defects were apparent in comparable proportions of patients who underwent scintigraphy after dobutamine and exercise stress $(65 \% v$ $73 \%$, NS). The sensitivity of exercise perfusion imaging among 34 patients with multivessel coronary disease (79\%) was comparable with the results of dobutamine perfusion imaging $(65 \%, \mathrm{NS})$. Of the 22 patients with single vessel coronary disease, exercise perfusion imaging identified 14 $(67 \%)$, and dobutamine scintigraphy identified $15(68 \%$, NS). The results of maximal and submaximal dobutamine testing were comparable in patients with multivessel and single vessel disease (table 6).

Patients with coronary disease undergoing submaximal dobutamine stress were less likely to have perfusion defects than those completing a maximal stress ( $58 \% v 76 \%$, NS), but this difference was less prominent than that with echocardiography. Similarly, $67 \%$ of 12 patients with coronary disease who exercised submaximally showed perfusion defects com-

Table 6 Perfusion abnormalities on scintigraphy during maximal and submaximal exercise and dobutamine stress testing

\begin{tabular}{lcrrl}
\hline & All patients & Maximal & Submaximal & $\begin{array}{l}\text { Maximal v } \\
\text { submaximal }\end{array}$ \\
\hline Sensitivity: & & Exercise & \\
Overall & $41 / 56(73 \%)$ & $33 / 44(75 \%)$ & $8 / 12(67 \%)$ & NS \\
Single vessel disease & $14 / 22(67 \%)$ & $12 / 18(67 \%)$ & $2 / 4(50 \%)$ & NS \\
Multivessel disease & $27 / 34(79 \%)$ & $21 / 26(81 \%)$ & $6 / 8(75 \%)$ & NS \\
Specificity & $21 / 30(70 \%)$ & $13 / 19(68 \%)$ & $8 / 11(73 \%)$ & NS \\
Accuracy & $62 / 86(72 \%)$ & $57 / 63(90 \%)$ & $16 / 23(70 \%)$ & 0.02 \\
Sensitivity: & & Dobutamine & & \\
Overall & $35 / 54(65 \%)$ & $16 / 21(73 \%)$ & $19 / 33(58 \%)$ & NS \\
Single vessel disease & $15 / 22(68 \%)$ & $8 / 10(80 \%)$ & $7 / 12(58 \%)$ & NS \\
Multivessel disease & $20 / 32(65 \%)$ & $8 / 11(73 \%)$ & $12 / 21(57 \%)$ & NS \\
Specificity & $19 / 28(68 \%)$ & $7 / 11(64 \%)$ & $12 / 17(71 \%)$ & NS \\
Accuracy & $54 / 82(66 \%)$ & $23 / 32(72 \%)$ & $29 / 50(58 \%)$ & NS \\
\hline
\end{tabular}

pared with $75 \%$ of the 44 who exercised maximally (NS). Indeed, only in the group undergoing submaximal dobutamine stress (table 3) was exercise significantly more sensitive than dobutamine stress perfusion imaging $(79 \% v$ $58 \%, P=0.02$ ).

EXTENT OF ISCHAEMIA AT DOBUTAMINE AND EXERCISE TESTING

Stress induced abnormalities in wall motion were apparent at both dobutamine and exercise stress testing in 28 patients with coronary disease. Even when both tests gave true positive results, the extent of wall motion abnormality at exercise $(34 \% \quad(18 \%)$ of left ventricular segments) exceeded the extent demonstrated as ischaemic using dobutamine echocardiography (15\% (8\%), $P<0.0001)$. Stress induced perfusion defects in the presence of coronary disease were present after both dobutamine and exercise stress in 33 patients. In this circumstance, the extent of perfusion defects at exercise $(29 \%(12 \%)$ of left ventricular segments) exceeded the extent of defects using dobutamine stress $(22 \%$ (11\%), $P=0.02$ ).

Dobutamine testing was also ineffective at identifying multivessel disease. At dobutamine echocardiography 22 patients with multivessel disease were identified as having coronary disease, but none was recognised as having stenoses in more than one vessel. In contrast, exercise identified 18 of 31 such patients $(58 \%, P<0.0001)$ as having multivessel disease. Likewise, only four out of 20 patients $(20 \%)$ identified as having coronary disease by dobutamine scintigraphy were recognised as having multivessel disease, compared with 15 out of 27 by exercise scintigraphy $(56 \%, P=0 \cdot 02)$.

\section{SPECIFICITY AND ACCURACY OF DOBUTAMINE}

AND EXERCISE STRESSES

No significant coronary stenoses were present in 30 patients. The specificity of electrocardiographic features of ischaemia with dobutamine and exercise were respectively $67 \%$ and $43 \%$ (NS). Normal results in echocardiograms on dobutamine testing were obtained in $25(83 \%)$ patients; normal results in peak exercise studies in $24(80 \%)$, and normal results in images after exercise in 26 (86\%). The specificity of echocardiography with maximal dobutamine stress ( $92 \%$ of 13 patients) and submaximal stress ( $76 \%$ of 17 patients) were comparable. As in the case of dobutamine stress, the level of exercise had no effect on the specificity of echocardiography, which was $79 \%$ in the 19 patients without disease who exercised maximally and $82 \%$ in the 11 who exercised submaximally. Similarly, at scintigraphy $21(70 \%)$ patients had normal results in exercise scans, and 19 out of 28 (68\%) had normal findings at dobutamine stress perfusion imaging. None of these findings differed significantly.

The accuracy of dobutamine stress electrocardiography (43\%) was significantly less than that of exercise electrocardiography $(65 \%$, $\mathbf{P}=0.0001)$. The accuracies of peak exercise 
(85\%) and postexercise echocardiography $(74 \%)$ were comparable (NS), and the accuracy of peak exercise exceeded that of dobutamine echocardiography at all concentrations $(64 \%, \quad P<0.0001)$, but not maximal dobutamine studies $(80 \%)$. The accuracy of scintigraphy with exercise (72\%) and dobutamine $(66 \%)$ were comparable.

\section{FEASIBILITY OF DOBUTAMINE AND EXERCISE} ECHOCARDIOGRAPHY

Stress echocardiography performed with dobutamine provided higher quality images than did exercise echocardiography. Of the 86 dobutamine echocardiograms, 20 were of $\mathrm{A}$ quality, 53 of B quality, 11 of $C$ quality, and two of $\mathrm{D}$ quality compared with three, 46,29 , and eight exercise echocardiograms respectively. Thus 73 dobutamine echocardiograms were of excellent or good quality (85\%) compared with 49 exercise echocardiograms (57\%, $\mathrm{P}<0.001)$.

The converse relation applied to diagnostic certainty, which was greatest with exercise echocardiography. Fifteen of the 86 dobutamine echocardiograms (17\%) were interpreted with a high level of confidence, compared with 32 exercise studies $(37 \%, \mathbf{P}=$ $0 \cdot 004)$. Moderate diagnostic confidence was attained in 47 dobutamine and 31 exercise echocardiograms, and low levels of diagnostic certainty were present in 24 dobutamine and 23 exercise echocardiograms.

The influence of image digitisation on accuracy was investigated by separate comparison of video tape and digitised images. Digital processing enhanced the accuracy of both dobutamine and exercise echocardiograms (64\% and $85 \%)$, compared with interpretation from video tape $(55 \%$ and $77 \%$ respectively). This benefit occurred mainly through a small improvement in sensitivity, both for dobutamine and exercise studies, at comparable levels of specificity.

\section{Discussion}

The accuracy of dobutamine echocardiography, together with its ease of performance, has led to enthusiasm for its general use rather than just in patients unable to exercise. Such a step mandates a direct comparison between the respective abilities of exercise and dobutamine echocardiography to induce ischaemia. Two important points arise in relation to this. Firstly, as the nature of the study group influences the results, the tests should be compared in the same population, under as close as possible to the same conditions. Secondly, because the influence of extraneous factors (such as drug treatment and submaximal testing) on the results may not be apparent in a selected study population, the appropriate environment for such a comparison is routine clinical practice. Indeed, our results indicate that the phenomena causing a submaximal dobutamine test are more common in routine clinical practice than in a controlled, study environment, being present in over $50 \%$ of patients.
ACCURACY OF DOBUTAMINE AND EXERCISE STRESS TESTING

Exercise electrocardiography is the cornerstone of stress testing, and the accuracy reported in recent meta-analyses ${ }^{2}$ is similar to our results. Dobutamine stress is a poor provocative test for the induction of electrocardiographic changes, and the dobutamine electrocardiogram is too insensitive to be used without imaging. ${ }^{20}$ The accuracy of dobutamine stress electrocardiography in our study is comparable to that reported previously. ${ }^{21}$

Exercise echocardiography before and after treadmill testing is now an established technique for the diagnosis of coronary disease; evidence from large studies show a sensitivity of $78-88 \%$ and a specificity of $80-100 \% .^{11-14}$ Though fewer data are available for exercising upright on a bicycle, ${ }^{15} 16$ they suggest greater sensitivity (87-91\%) with lower specificity (78-80\%) and are concordant with our results. Abnormal regional function may resolve early after exercise, so imaging at peak exercise may identify ischaemia more readily than after exercise. ${ }^{22}$ This is the most likely explanation of the greater sensitivity of bicycle echocardiography. The lower specificity of peak imaging may reflect suboptimal image quality and artefacts introduced by a moving body and heart. Independent of the nature of exercise, sensitivity reflects the nature of the population studied, with larger numbers of patients with previous myocardial infarction and multivessel coronary disease tending to increase the sensitivity, ${ }^{12}$ and patients with moderate stenoses and submaximal exercise capacity tending to reduce the sensitivity of post-treadmill echocardiography. ${ }^{14}$ The adverse influence of submaximal exercise on the sensitivity of bicycle echocardiography was confirmed in our study.

The nature of the study group has similarly proved important to the results of dobutamine echocardiography, the sensitivity of which has ranged from $54 \%$ to $66 \%$ and the specificity from $96 \%$ to $100 \%$; these results encompass those of our study. In addition to the factors causing variability in the accuracy of exercise echocardiography, the greater heterogeneity of results from dobutamine echocardiography testifies to other potential extraneous influences. The frequency of side effects requiring test termination has varied from $3 \%$ to $20 \%$, reflecting both different study populations as well as different criteria for stopping the test. ${ }^{4-823}$ Ischaemia is less likely in tests completed at a lower dose than in those in which high doses are attained. ${ }^{1021}$ The exclusion of patients undergoing submaximal stress in some previous studies 5 may explain some of the higher levels of sensitivity previously recorded. The influence of $\beta$ adrenoceptor blockade on the results of dobutamine echocardiography in previous studies may have been attenuated by higher prevalence of coronary disease in patients treated with these drugs, ${ }^{6}$ as well as failure to address the timing of the dose before the test (and hence the extent of $\beta$ blockade). Submaximal testing 
(due either to $\beta$ blockade or termination of the test at low dose) has been shown in this study to reduce the ischaemic potential of dobutamine stress.

The results of myocardial perfusion scintigraphy at dobutamine stress ${ }^{910}$ parallel those reported using exercise, ${ }^{24}$ a finding supported by our results. The performance of submaximal exercise limits the sensitivity of thallium imaging, ${ }^{25}$ but the size of the submaximal stress group was too small to repeat this analysis with perfusion imaging. While exercise and dobutamine stress have not been compared in the same patients, previous direct compari sons of exercise and non-exercise (dipyridamole) perfusion imaging have provided comparable results. ${ }^{26}$

\section{PREVIOUS DIRECT COMPARISONS OF THE} ISCHAEMIC POTENTIAL OF DOBUTAMINE AND EXERCISE STRESS

Few data comparing dobutamine with exercise testing in the same patients are available. In a previous direct comparison of electrocardiographic results, Mazieka et al found that dobutamine electrocardiography was less sensitive than exercise echocardiography $(47 \% v$ $72 \%$, NS). ${ }^{21}$ Two previous studies have directly compared the ability of exercise performed in the supine position and dobutamine to provoke abnormal left ventricular function in the same patients. Hoffman et al found comparable levels of sensitivity (respectively $80 \%$ and $79 \%$ ) and specificity (respectively $87 \%$ and $81 \%$ ) with exercise and dobutamine. ${ }^{27}$ They, however, discontinued treatment for 24 hours in all patients, none of whom had suboptimal studies. Cohen et al reported a sensitivity of $76 \%$ with exercise and $86 \%$ with dobutamine echocardiography, again using supine bicycle stress testing in patients who were not receiving treatment. ${ }^{28}$ Our results are concordant with these studies to the extent that the results of maximal dobutamine and exercise echocardiography were comparable. Our results also emphasise, however, the importance of clinical factors, which are often not apparent under the supervised environment of a study, though they may compromise the clinical sensitivity of either test, particularly dobutamine echocardiography.

ABIIITY OF EXERCISE AND DOBUTAMINE STRESSES TO EVALUATE EXTENT OF CORONARY DISEASE

In addition to diagnosing coronary artery disease, non-invasive tests may be used to assess the functional extent of ischaemia. In our study dobutamine stress was ineffective for this purpose, underestimating the extent of ischaemia (and to a lesser extent, perfusion abnormality) shown in exercise stress testing. Although the recognition of multivessel disease in patients with positive tests is problematic for all stress imaging strategies (because stress may be limited by ischaemia in one area before it becomes apparent in another), this was a particular shortcoming of dobutamine stress in combination with both echo- cardiography and scintigraphy. Finally, electrocardiographic changes with stress that reflect the severity of coronary disease are of prognostic value ${ }^{29}$ and occur significantly less often with dobutamine than with exercise stress testing.

\section{CLINICAL IMPLICATIONS - WHICH IS THE} OPTIMAL STRESS IN ACTIVE PATIENTS?

Our results indicate that exercise imposes a greater workload on the heart than dobutamine stress. Exercise is therefore a more potent precipitant of ischaemia than dobutamine, while this difference is less prominent for perfusion heterogeneity. In patients who are able to exercise maximally this translates into greater sensitivity for exercise than dobutamine electrocardiography and echocardiography. Although the ability of any technique to identify ischaemia is compromised by submaximal stress, this occurred more frequently with dobutamine testing, which was also more vulnerable to this effect than exercise. Thus, patients undergoing dobutamine echocardiography are likely to have results comparable with those they would have with exercise only if they undergo maximal dobutamine stress. Indeed, even in those patients with concordant positive results, the number of abnormal segments at either echocardiography or scintigraphy was larger after exercise than dobutamine stress, suggesting a greater extent of ischaemia. Moreover, while dobutamine echocardiography offers benefits in terms of imaging performance and quality, this is balanced by greater difficulty in interpretation because of more localised and milder abnormalities in wall motion.

The selection of dobutamine stress has the obvious disadvantage of not obtaining data on exercise capacity in patients who can exercise. The dobutamine protocol cannot offer information about exercise capacity and the haemodynamic response to stress, which have prognostic significance. In clinical terms the development of ischaemia at peak doses of dobutamine is less meaningful clinically than development of ischaemia at a workload which can be equated to the work expenditure required for daily activities.

\section{CONCLUSIONS}

Our results suggest that earlier reports of high sensitivity using dobutamine echocardiography are difficult to replicate in routine clinical practice because the dobutamine test is more vulnerable to extrinsic influences than is exercise stress. There is no good reason to replace exercise with dobutamine stress testing in patients who can exercise maximally. When dobutamine echocardiography is selected in preference to exercise echocardiography in routine clinical practice patients should stop angina treatment and every effort should be made to complete the dobutamine protocol. Results showing no inducible ischaemia in patients who have not completed the protocol should be identified as non-diagnostic rather than negative. 
1 Corday E, Hajduczki I, O'Byrne GT, Kar S, Areeda J, Corday SR. Echocardiographic criteria to distinguish reversible from irreversible myoca

2 Detrano R, Froelicher VF. Exercise testing: uses and limitations considering recent studies. Prog Cardiovasc Dis 1988;31:173-204.

3 Feigenbaum $\mathrm{H}$. Exercise echocardiography. Fournal of the American Society of Echocardiography 1988;1:161-6.

4 Sawada SG, Segar DS, Ryan T, Brown SE, Dohan AM, Williams $R$, et al. Echocardiographic detection of coronary artery disease during dobutamine infusion. nary artery disease during

5 Cohen JL, Greene TO, Ottenweller J, Binenbaum SZ, Wilchfort SD, Kim CS. Dobutamine digital echocardiography for detecting coronary artery disease. $\mathrm{Am} \mathcal{J}$

Salual 1991;67:1311-8.

Salustri A, Fioretti PR, Pozzoli MMA, McNeill AJ, Roelandt JR. Dobutamine stress echocardiography: its role in the diagnosis of coronary artery disease. Eur Heart f 1992;13:70-7.

7 Marcowitz P, Armstrong WF. Accuracy of dobutamine stress echocardiography in detecting coronary artery disease. Am $\mathcal{F}$ Cardiol 1992;69:1269-73.

8 Previtali M, Lanzarini L, Ferrario M, Tortorici M, Mussini A, Montemartini C. Dobutamine versus dipyridamole echocardiography in coronary artery disease. Circulation 1991;83(suppl III):27-31.

9 Pennell DJ, Underwood SR, Swanton RH, Walker JM, Ell PJ. Dobutamine thallium myocardial perfusion tomography. 7 Am Coll Cardiol 1991;18:1471-9.

10 Marwick T, D'Hondt AM, Baudhuin T, Willemart B, Wijns W, Detry JM, et al Optimal use of dobutamine stress for the detection and evaluation of coronary artery disease: combination with echocardiography, scintigraphy or both? $₹ \mathrm{Am}$ Coll Cardiol 1993;22:159-67.

11 Armstrong WF, O'Donnell J, Dillon JC, McHenry PL, Morris SN, Feigenbaum H. Complementary value of two-dimensional exercise echocardiography to routine two-dimensional exercise echocardiography to rout

12 Armstrong WF, O'Donnell J, Ryan T, Feigenbaum $H$. Effect of prior myocardial infarction and extent and Effect of prior myocardial infarction and extent and
location of coronary disease on accuracy of exercise location of coronary disease on accuracy of exercise

13 Ryan T, Vasey CG, Presti CF, O'Donnell J, Feigenbaum H, Armstrong WF. Exercise echocardiography: detection of coronary artery disease in patients with norma left ventricular wall motion at rest. $\mathcal{f} \mathrm{Am}$ Coll Cardio 1988;11:993-9.

14 Marwick T, Nemec J, Pashkow FJ, Stewart WJ, Salcedo EE. Accuracy and limitations of exercise echocardiography in a routine clinical setting. $\mathcal{f} \mathrm{Am}$ Coll Cardiol 1992;19:74-81.

15 Mertes H, Erbel R, Nixdorff U, Mohr-Kahaly S, Wolfinger D, Meyer J. Stress echocardiography: a sensitive method for the detection of coronary artery disease. Herz 1991;16:355-66.

16 Ryan T, Segar DS, Sawada SG, et al. Detection of coronary artery disease with upright bicycle exercise echocar- diography. Fournal of the American Society of Echocardiography 1993;6:186-97.

17 Detry JM, Robert A, Luwaert RJ, et al. Diagnostic value of computerized exercise testing in men without previous myocardial infarction. A multivariate compartmental and probabilistic approach. Eur Heart $\mathcal{F}$ 1985;6:227-38.

18 Broderick T, Sawada S, Armstrong WF, et al. Improvement in rest and exercise-induced wall motion abnormalities after coronary angioplasty: an exercise echocardiographic study. $\mathcal{f} \mathrm{Am}$ Coll Cardiol 1990;15: 591-9.

19 Taillefer R, Gagnon A, Laflamme L, Grégoire J, Léveillé J, Taillefer R, Gagnon A, Laflamme L, Gregoire J, Leveille J,
Phaneuf DC. Sameday injections of Tc-99m methoxy isobutyl isonitrile (hexamibi) for myocardial tomoisobutyl isonitrile (hexamibi) for myocardial tomographic imaging: comparison between rest-stress and
stress-rest injection sequences. Eur $f$ Nucl Med 1989; stress-rest inje.

20 Mairesse G, Marwick T, D'Hondt AM, Wijns W, Melin JA, Detry JM. How reliable is the dobutamine stress ECG for the diagnosis of coronary disease? A receiver operating curve analysis [abstract]. I Am Coll Cardiol 1993;21:174A.

21 Mazieka PK, Nadazdin A, Oakley CM. Dobutamine stress echocardiography for detection and assessment of coronary artery disease. $\mathcal{F} \mathrm{Am}$ Coll Cardiol 1992;19: 1203-11.

22 Presti CF, Armstrong WF, Feigenbaum H. Comparison of echocardiography at peak exercise and after bicycle exercise in evaluation of patients with known or suspected coronary artery disease. Fournal of the American Society of Echocardiography 1988;1:119-26.

23 Mertes H, Sawada SG, Ryan T, et al. Symptoms, adverse effects, and complications of dobutamine stress echocardiograpy Experience in 1118 patients. Circulation 1993;88:15-9.

24 Kotler TS, Diamond GA. Exercise thallium-201 scintigraphy in the diagnosis and prognosis of coronary artery
disease. Ann Intern Med 1990;113:684-702.

25 Iskandrian AS, Heo J, Kong B, Lyons E. Effect of exercise Iskandrian AS, Heo J, Kong B, Lyons E. Effect of exercise
level on the ability of thallium-201 tomographic imaging in detecting coronary artery disease: analysis of 461 in detecting coronary artery disease: analysis

26 Timmis AD, Lutkin JE, Fenney LJ, et al. Comparison of dipyridamole and treadmill exercise for enhancing thallium-201 perfusion defects in patients with coronary lium-201 perfusion defects in patients
artery disease. Eur Heart $f$ 1980;1:275-80.

27 Hoffman $R$, Lethen $H$, Kleinhaus $E$, Weiss $M$, Flachskampf FA, Hanrath P. Comparative evaluation of bicycle and dobutamine stress echocardiography with perfusion scintigraphy and bicycle electrocardiogram for evaluation of coronary artery disease. $\mathrm{Am} \mathcal{f}$ Cardiol 1993;72:555-9.

28 Cohen JL, Duvvuri S, George A, et al. Dobutamine versus exercise digital echocardiography for detecting coronary artery disease [abstract]. F Am Coll Cardiol 1992;19:32A.

29 Chaitman BR. The changing role of the exercise electrocardiogram as a diagnostic and prognostic test for 1986;8:1195-1210. 
cardiac rhabdomyomata and fetal hydrops in a premature infant with tuberous sclerosis. $f$ Paediatr Child Health 1992;28:260-2.

16 Sauter R, Hummler H, Klemm I. Neonatal heart tumor Early symptom of tuberous sclerosis. Monatsschr Kinderheilkd 1992:140:21-3.

17 Birnbaum SE, McGahan JP, Janos GG, Meyers M. Fetal tachycardia and intramyocardial tumors. $7 \mathrm{Am}$ Coll tachycardia and intram
Cardiol 1985;6:1358-61.

18 Platt LD, Devore GR, Horenstein J, Pavlova Z, Kovacs B, Falk RE. Prenatal diagnosis of tuberous sclerosis: the use of fetal echocardiography. Prenat Diagn 1987;7:407-11.

19 Crawford DC, Garret C, Tynan M, Neville BG, Allan LD. Cardiac rhabdomyomata as a marker for the antenatal detection of tuberous sclerosis. $\mathcal{F}$ Med Genet 1983;20 303-12.

20 Riikonen $\mathbf{R}$, Simell $O$. Tuberous sclerosis and infantile spasms. Dev Med Child Neurol 1990;32:203-9.

21 Manetti A, De Simone L, Favilli S, et al. Echo- cardiographic study of heart rhabdomyoma in tuberous sclerosis. G Ital Cardiol 1989;19:319-23.

22 Konkol RJ, Walsh EP, Power T, Bresnan MJ. Cerebral embolization resulting from an intracardiac tumor in tuberous sclerosis. Pediatr Neurol 1986;2:108-10.

23 Gomez MR. Strokes in tuberous sclerosis: are rhabdomyomas a cause? Brain Dev 1989;11:14-9.

24 Farooki ZQ, Ross RD, Paridon SM, Humes RA, Karpawich PP, Pinsky WW. Spontaneous regression of cardiac rhabdomyoma. Am f Cardiol 1991;67:897-900.

25 Alkalay AL, Ferry DA, Lin B, Fink BW, Pomerance JJ. Spontaneous regression of cardiac rhabdomyoma in tuberous sclerosis. Clin Pediatr 1987;26:532-5.

26 Houser S, Forbes N, Stewart S. Rhabdomyoma of the heart: a diagnostic and therapeutic challenge. $A n n$ Thorac Surg 1980;29:373-7.

27 Pesonen $\mathrm{E}$, Leijala $\mathrm{M}$, Järvenpää $\mathrm{AL}$ Teramo $\mathrm{K}$ Cardiac rhabdomyomas in a newborn baby. Acta Paediatr Scand 1985;74:824-7.

\section{CORRECTION}

Table 2 Electrocardiographic responses during maximal ( $P$ value) and submaximal exercise and dobutamine stress testing

\begin{tabular}{lccrl}
\hline & All patients & Maximal & Submaximal & \multicolumn{1}{c}{$\begin{array}{l}\text { Maximal vs } \\
\text { submaximal }\end{array}$} \\
\hline Sensitivity & $43 / 56(77 \%)$ & $39 / 44(89 \%)$ & $4 / 12(33 \%)$ & 0.0003 \\
Specificity & $13 / 30(43 \%)$ & $4 / 19(21 \%)$ & $9 / 11(82 \%)$ & 0.002 \\
Accuracy & $56 / 86(65 \%)$ & $43 / 63(68 \%)$ & $13 / 23(57 \%)$ & NS \\
& & Dobutamine & & \\
Sensitivity & $18 / 56(32 \%)$ & $13 / 22(59 \%)$ & $5 / 34(15 \%)$ & $<0 \cdot 001$ \\
Specificity & $19 / 30(63 \%)$ & $7 / 13(54 \%)$ & $12 / 17(71 \%)$ & NS \\
Accuracy & $37 / 86(43 \%)$ & $20 / 35(57 \%)$ & $17 / 51(33 \%)$ & 0.02 \\
\hline
\end{tabular}

Table 6 Perfusion abnormalities on scintigraphy during maximal and submaximal exercise and dobutamine stress testing

\begin{tabular}{lcccl}
\hline & All patients & Maximal & Submaximal & $\begin{array}{l}\text { Maximal vs } \\
\text { submaximal }\end{array}$ \\
\hline Sensitivity & & Exercise & \\
Overall & $41 / 56(73 \%)$ & $33 / 44(75 \%)$ & $8 / 12(67 \%)$ & NS \\
Single vessel disease & $14 / 22(64 \%)$ & $12 / 18(67 \%)$ & $2 / 4(50 \%)$ & NS \\
Multivessel disease & $27 / 34(79 \%)$ & $21 / 26(81 \%)$ & $6 / 8(75 \%)$ & NS \\
Specificity & $21 / 30(70 \%)$ & $13 / 19(68 \%)$ & $8 / 11(73 \%)$ & NS \\
Accuracy & $62 / 86(72 \%)$ & $46 / 63(73 \%)$ & $16 / 23(70 \%)$ & NS \\
& & Dobutamine & & \\
Sensitivity & & & \\
Overall & $35 / 54(65 \%)$ & $16 / 21(76 \%)$ & $19 / 33(58 \%)$ & NS \\
Single vessel disease & $15 / 22(68 \%)$ & $8 / 10(80 \%)$ & $7 / 12(58 \%)$ & NS \\
Multivessel disease & $20 / 32(63 \%)$ & $8 / 11(73 \%)$ & $12 / 21(57 \%)$ & NS \\
Specificity & $19 / 28(68 \%)$ & $7 / 11(64 \%)$ & $12 / 17(71 \%)$ & NS \\
Accuracy & $54 / 82(66 \%)$ & $23 / 32(72 \%)$ & $31 / 50(62 \%)$ & NS \\
\hline
\end{tabular}

Comparative ability of dobutamine and exercise stress in inducing myocardial ischaemia in active patients. $T H$ Marwick, $A M D^{\prime} H o n d t, G H$ Mairesse, $T$ Baudhuin, $W$ Wijns, $\mathcal{f} M$ Detry, $\mathcal{f} A$ Melin.

The authors of this article miscalculated some percentages in tables 2 and 6 ( $B r$ Heart $\mathcal{f}$ 1994;72:31-8). Their corrected versions of these tables appear alongside. 